\title{
The Technology Research on License Plate Location and Character Segmentation
}

\author{
Yu-Chun Zhang \\ School of science, ShenYang Ligong University, Shenyang , China.110159 \\ awangkaixiang004@163.com
}

Keywords: License plate location, Image segmentation, Contour tracing, Character segmentation

\begin{abstract}
License plate identification technology is an important link of license plate recognition system, the pretreatment result will affect the subsequent effect of license plate recognition. This article research license plate location and character segmentation technology, proposing an improved Otsu algorithm and projection method to binary processing and location of the license plate, improve the accuracy of the threshold and location accuracy. Based on template method to segment the characters of license plate, horizontal scanning the characters after vertical segmentation of the license plate, finally realize the horizontal partition and vertical division. They all get good effects.
\end{abstract}

\section{The Binary Algorithms of License Plate Image}

The binary image algorithm of license plate based on gray image is selected an appropriate threshold to separate license plate background and characters effectively. This paper proposes an improved Otsu algorithm for binary algorithm of image processing, automatic access to the threshold.

\section{The Otsu Algorithms}

Using $\mathrm{D}$ as the classification of the signs, less than $\mathrm{D}$ for the class of $\mathrm{C} 1$, more than $\mathrm{D}$ for the class of $\mathrm{C}_{2}$. Looking through the array of histogram and cumulative the sum of gray level denoted as $\mathrm{n} 1$. Where $\mathrm{W}$ is the gray average and $\mathrm{U}$ is the weighted average of gray. Where

$$
\mathrm{p}=\mathrm{x} *(\mathrm{k}-\mathrm{u}) *(\mathrm{k}-\mathrm{u})
$$

to cumulative sum of $\mathrm{P}$. Using $\mathrm{u} 1$ as the weighted average of $\mathrm{C} 1$, where

$$
\mathrm{n} 2=\mathrm{n}-\mathrm{n} 1
$$

to cumulative the sum of P1.

$$
\mathrm{p} 1=\mathrm{x} *(\mathrm{k}-\mathrm{u} 1) *(\mathrm{k}-\mathrm{u} 1)
$$

To place the two variance ratio in the array $c[d]$, where

$$
[\mathrm{d}]=(\mathrm{w} * \mathrm{w} 1 *(\mathrm{u}-\mathrm{u} 1) *(\mathrm{u}-\mathrm{u} 1)) /(\mathrm{p}+\mathrm{p} 1)
$$

The value of $\mathrm{D}$ in circulation increase one every time and every calculated variance ratio will be stored in the array c[d]. The gray array is divided into two parts, the part of 0 to the 150 and the other part of 150 to 255 . Take 20 for threshold in the first part, 20 times per cycle to assign gray accumulation value of Max to $\mathrm{m} 2$, where

$$
\mathrm{s} 1=\mathrm{g}-10
$$

In the second part take 20 for threshold, 20 times per cycle to assign gray accumulation value of Max to m3, where

$$
\begin{aligned}
& \mathrm{s} 2=\mathrm{gt}, \\
& \mathrm{mid}=(\mathrm{s} 1+\mathrm{s} 2) / 2
\end{aligned}
$$

make the mid for the final thresholds of Otsu algorithm. One by one scan the pixel and call the rgbgray function to gray each point. According to the threshold of mid we obtained by Otsu algorithm, if the gray value is less than mid, the RGB value is set to $(0,0,0)$. If the gray value greater than mid, the point of the RGB is set to a value of $(255,255,255)$, so that we achieve the local binarization algorithms processing. 


\section{The Improved Otsu Algorithm}

The improved Otsu algorithm is to obtain a basic threshold $t$ and take the image segmentation, So the original image can be divided into two parts $\mathrm{C} 1$ and $\mathrm{C} 2$, The one part of the gray value is greater than $t$, another part of gray value is less than $t$. To calculate the maximum between-cluster variance on $\mathrm{C} 1$ and $\mathrm{C} 2$, Finally found the best image threshold t, the ratio of the biggest variance is the best threshold value of image. Let the total number of pixels of class C1 is W1and the total number of pixels of class $\mathrm{C} 2$ is W2, the pixel gray value of them are M1 and M2,where

$$
\begin{aligned}
& \sigma_{B}^{2}=W_{1} W_{2}\left(M_{1}-M_{2}\right)^{2} \\
& \sigma_{A}^{2}=W_{1} \sigma_{1}^{2}+W_{2} \sigma_{2}^{2}
\end{aligned}
$$

Reading the license plate area that has been located and take the gray level histogram processing.Then using the improved Otsu algorithm to obtain the threshold, the threshold is accurate. According to the threshold, we binary processing the pixels one by one and then output the binary image result.

\section{License Plate Location Technology of Gray Image}

License plate location is the most important part of the whole license plate recognition system, In the algorithm based on gray image, this paper using the projection method for license plate location. in horizontal, we use first-order difference operation:

$g(i, j)=f(i, j)-f(i, j+1)$

In the vertical scanning process, we are still using first-order difference operation:

$g(i, j)=f(i, j)-f(i+1, j)$

when there is a straight line from the horizontal or vertical direction through the license plate area, alternating black and white pixels in the area will produce dramatic changes, because of the existence of license plate characters.

\section{License Plate Character Segmentation Based on Template Method}

Firstly, to read the bitmap after locate the license plate (the four vertex coordinates are the most important aspect of the license plate). We according to the license plate of the prior knowledge comprehensive template method for license plate character segmentation, the vertical segmentation of license plate characters based on horizontal scan, achieve the goal of horizontal split.

\section{The Horizontal Character Segmentation}

Reading the bitmap data of the positioned license plate. Defining the local variables ste, where ste=(right-left) $/ 7$ as the empirical value 。 "Right" is the abscissa of the plate right boundary and "left" is the abscissa of the plate left boundary .Let

c9=(right-left) $* 2 / 15$

where c9 is the experience value of the character position,

fanwei=(right-left) $/ 25+0.5$

According to the piror knowledge of the license plate

fengepoint $=\mathrm{left}+2 * \mathrm{c} 9$

is the segmentation point coordinates between second characters and third characters. To segment the rest of the characters in order, the coordinates are right-c9, right- $2 *_{\mathrm{c}} 9$, right- $3 *_{\mathrm{c}} 9$, right $-4 * c 9$, right $-5 * c 9$.

We through numberwhite function to make line coordinates more optimized, numberwhite function returns a list of white pixel values. According to the previously "fanwei" variables, If the values of a column of white pixel are less than the current column, the abscissa of the column is 
assigned to the current line coordinates.By calling the moveto(fenge[i],top), lineto(fenge[i],down) function division, variable (I) increasing from 0 to 6 in order. This completes the vertical segmentation process of license plate characters.

\section{The Horizontal character segmentation}

Determine the line at the top of the characters, first define Boolean variable jjjj is true for judging whether reach the boundary of character. To scan the longitudinal coordinate in decrease from $j=($ top + down $) / 2$, transverse scoper is the abscissa of two adjacent line, If encounter white pixels, the Boolean variable JJJJ assignment to false. Until the JJJJ evaluates to true that has reached the character boundary, returns the current y coordinate value $j$. The average value of the horizontal split at the top of the characters is the average value.

Repeat the above process and scan each adjacent respectively line . To compute the 7 line coordinates and return them to coordinate, take the maximum and the minimum values form the seven coordinates, remove the last value then computer the average of the remaining coordinates. The average is the horizontal coordinates of the characters at the bottom.

\section{The experiment results}

Using the improved Otsu algorithm to obtain the threshold, the threshold is accurate. According to the threshold, we binarization processing the pixels one by one and then output the binary result.

\section{A.B583L A.B583L A.P4602 AP4602}

Fig. 3 binary image

We use the previously mentioned methods for horizontal and vertical scanning the area. The license plate can be precise positioning. To normalization process the license plate area that have determine the boundaries. The result of the gray image show in the figure 6 to figure 8 .

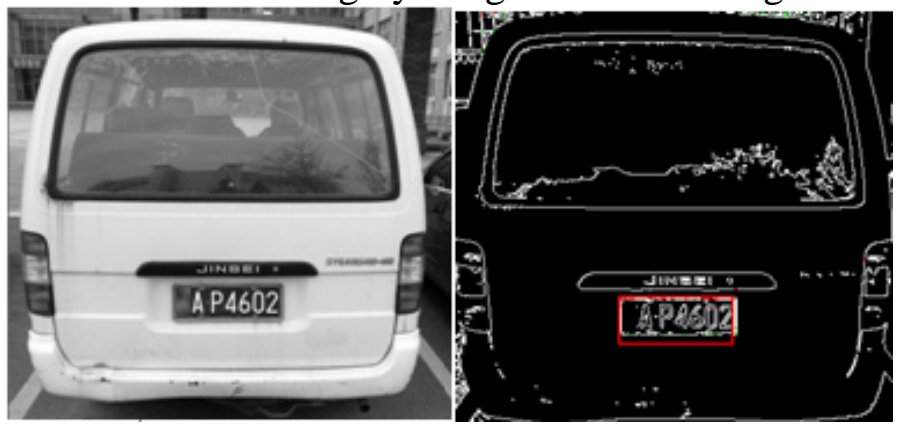

Fig. 4 Gray image Fig. 5The coarse position of the license plate

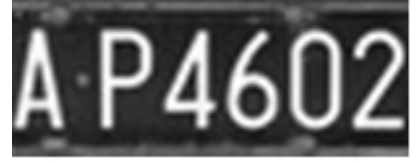

Fig. 6 The accurate position of the license plate

We according to the license plate of the prior knowledge comprehensive template method for license plate character segmentation, the vertical segmentation of license plate characters based on horizontal scan, achieve the goal of horizontal split. This method is simple and rapid. The result of license plate character segmentation show in figure 9 


\section{A.4M819 A:4M8119 A.P4602 A.P4602}

Fig. 7 character segmentation

\section{Summary}

This paper has studied the license plate localization and license plate character segmentation technology. Using an improved Otsu algorithm to binary image processing the image, obtain the required threshold automatically, then output the result of binary, improve the accuracy of the threshold. The license plate character segmentation based on template method, scaning the license plate character Horizontal based on the vertical segmentation, Realize the horizontal partition and vertical division.

\section{References}

[1]Xu Z..Rotation-invariant text classification using featuredistributions ， Pattern Recognition , 2000, vol.33:43-52

[2]Alessandro Vinciarelli.A Survey on off-line Cursive word Recognition[J].Pattern Recognition 20 02(35):1433-1446

[3] H.Erdinc.Kocer, K.Kursat Cevik Artificial neural networks based vehicle license plate recognition. Procedia Computer Science. 2011(3)1033-1037

[4] ZhengDN,ZhaoYN,Wang JX.An efficient method of license Plate location. Pattern Recognition . 2005

[5] Nicolas Thome, Antoine Vacavant, Lionel Robinault,Serge Miguet, A cognitive and video-based approach for multinational License Plate Recognition.Machine Vision and Applications(2011) 22:389-407 